\title{
Histoplasmosis of the central nervous system
}

\author{
V Tan, P Wilkins, S Badve, M Coppen, S Lucas, R Hay, F Schon
}

\begin{abstract}
Histoplasma capsulatum infection of the central nervous system is extremely rare in the United Kingdom partly because the organism is not endemic. However, because the organism can remain quiescent in the lungs or the adrenal glands for over 40 years before dissemination, it increasingly needs to be considered in unexplained neurological disease particularly in people who lived in endemic areas as children. In this paper a rapidly progressive fatal myelopathy in an English man brought up in India was shown at necropsy to be due to histoplasmosis. The neurological features of this infection are reviewed.
\end{abstract}

Histoplasma capsulatum is a dimorphic fungus that exists as a soil saprophyte in hyphal phase but forms small yeast like cells within the human reticuloendothelial system. Infection with this fungus is endemic in the eastern and central states of the USA and in some 60 temperate and tropical countries throughout the world including parts of India and South East Asia. In the United Kingdom, however, histoplasmosis infection remains extremely rare. The disease is not endemic in the UK and all cases are thought to be imported. ${ }^{12}$ Slowly evolving, chronic disseminated, forms of histoplasmosis, however, present many years after a patient has left an endemic area.

Until recently, almost all cases worldwide occurred in otherwise healthy individuals and consisted of an acute or chronic pulmonary disease resembling tuberculosis. Extrapulmonary histoplasmosis was uncommon and occurred most often in infant, elderly and immunocompromised patients. Disseminated disease is now, however, being seen more frequently because of the increasing prevalence of acquired immunodeficiency syndrome. ${ }^{5}$

Histoplasmosis of the CNS is rare but occurs in up to $50 \%$ of cases of generalised dissemination. ${ }^{6-7}$ In some cases the "walled off' foci of infection are found in the lungs but an indolent infection can also reside within the adrenal glands, presenting many years later. Adrenal gland involvement often remains inapparent until necropsy examination. ${ }^{8}$ Shapiro $e t$ al in $1955^{7}$ suggested that CNS histoplasmosis should be divided into three main forms by analogy with tuberculosis, namely: miliary granulomas; chronic basal lepto-meningitis and solitary parenchymal granulomas.
This report involves a patient with histoplasma myelopathy in association with multiple CNS histoplasmomata in whom the infection presumably remained quiescent in the adrenal glands and possibly elsewhere for at least 38 years. No cases of CNS histoplasmosis have been reported from the United Kingdom (UK) in recent years.

\section{Case report}

The patient was a 72 year old white retired local government official who was born in Bangalor in India of two English parents. $\mathrm{He}$ travelled extensively in India and Burma as a child with his father who was in the British Army in India. He returned to the UK in 1950 and did not subsequently travel outside Europe. In January 1989 he developed a dry cough, dyspnoea, ankle swelling, malaise, anorexia and weight loss. Examination revealed bilateral inspiratory creptitations and hepatosplenomegaly.

Pulmonary function tests showed a restrictive defect and chest $x$ ray interstitial diffuse shadowing. Liver function tests revealed an elevated bilirubin and alkaline phosphatase with negative serology for Hepatitis B virus. Liver biopsy showed changes of micronodular cirrhosis of undertermined aetiology. Transbronchial lung biopsy confirmed interstitial fibrosis consistent with fibrosing alveolitis.

A diagnosis of fibrosing alveolitis and cryptogenic cirrhosis was made and the patient was treated with prednisolone $50 \mathrm{mgm}$ per day. A month later there was a marked improvement in his symptoms and signs. He remained well over the next four months apart from steroidinduced diabetes mellitus which was controlled on oral hypoglycaemia agents. The steroid dose was gradually reduced to $10 \mathrm{mgm}$ per day.

In November 1989 he was readmitted to hospital with a three day history of progressive weakness and numbness in both legs associated with urinary incontinence. On examination there was a flaccid paraparesis and generalised areflexia. Myelography was unremarkable as was the cerebrospinal fluid (46 red blood cells $\times 10^{6} /, 0$ white blood cells, glucose $3.4 \mathrm{mmol} / 1$, protein $0.6 \mathrm{mmol} / \mathrm{l}$ ). Nerve conduction studies showed evidence of a generalised sensory motor neuropathy with significant slowing of conduction velocities. Over the subsequent four weeks progressive neurological deterioration occurred to a complete 


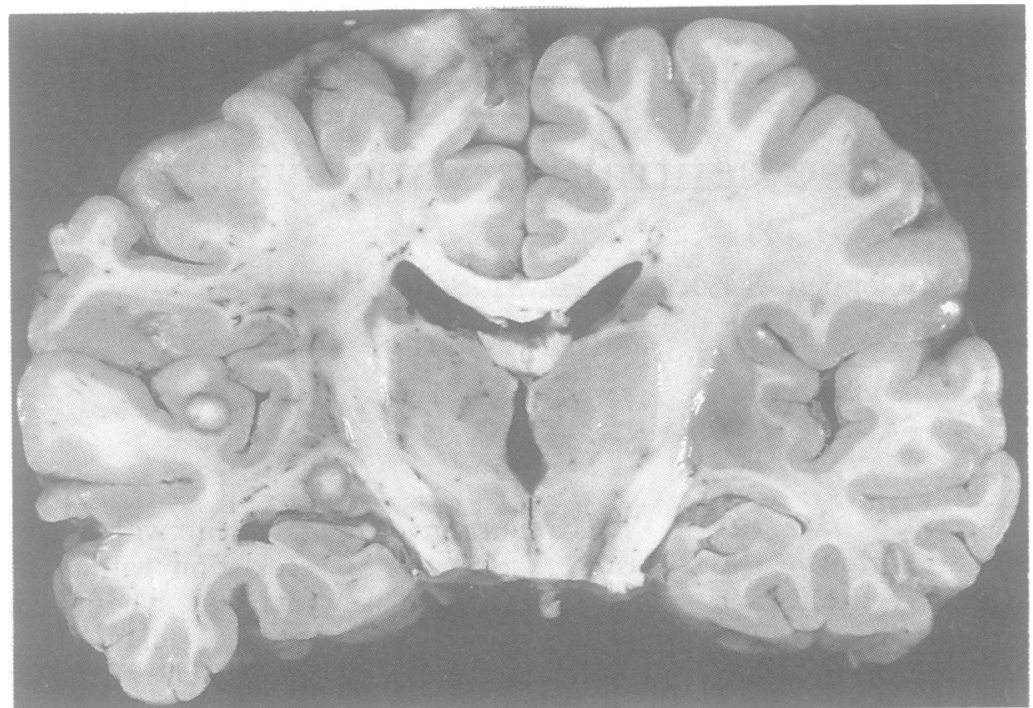

Figure 1 Coronal Section through the hemisphere showing discrete nodules in the left superior temporal gyrus and the left lateral geniculate body.

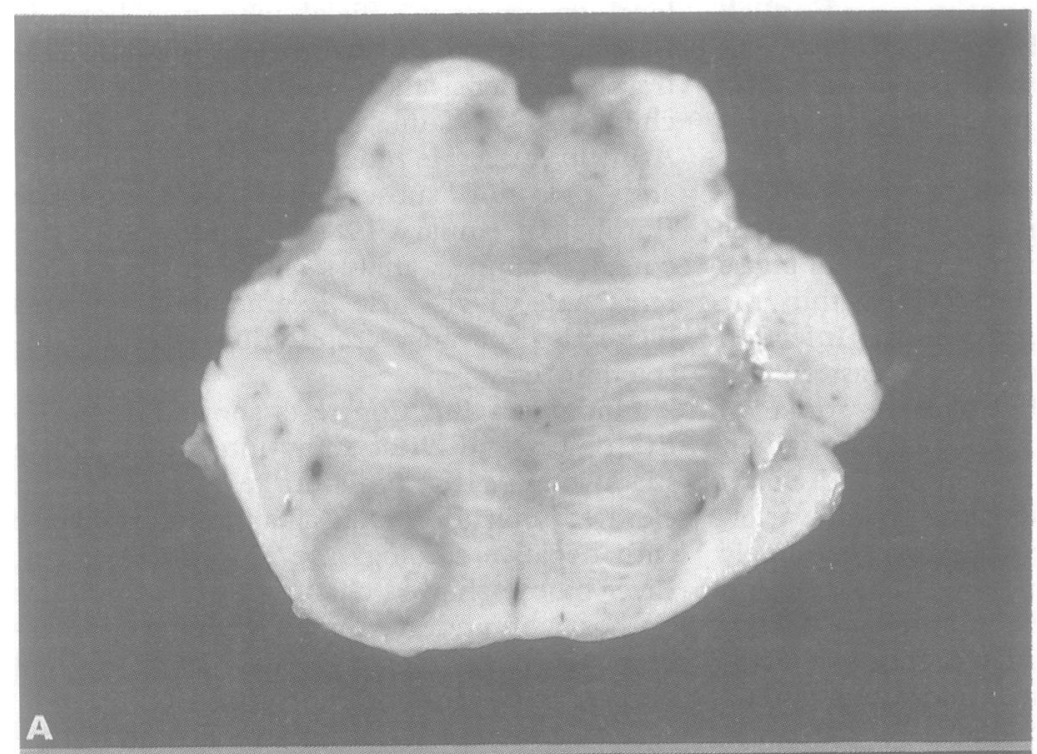

Figure 2 (a) Transverse section through the pons showing a $4 \mathrm{~mm}$ diameter nodule right anterior region. (b) Transverse section through mid lumbar spinal cord showing a $4 \mathrm{~mm}$ diameter nodule occupying the bulk of the cord. paraplegia, his speech became dysarthric and weakness developed in his right arm. His reflexes remained absent and both plantar responses became extensor. All sensory modalities were reduced in both legs with a sensory level at the tenth thoracic dermatome. Urinary retention necessitated catheterisation.

At this stage the patient was assumed to have a severe radiculomyelopathy of uncertain aetiology. A CT brain scan without contrast enhancement and an MRI of the spinal cord also without enhancement showed no significant abnormalities. No specific serological tests for histoplasmosis or human immunodeficiency virus (HIV) infections were carried out. Six weeks after developing his neurological condition he developed a chest infection and despite appropriate treatment died of a cardiopulmonary arrest.

PATHOLOGY

General

The heart contained an old myocardial infarct and occlusive atherosclerosis. Both lungs were moderately congested but were otherwise unremarkable. The spleen was enlarged and congested. The liver showed a mixed micro and macro-nodular cirrhosis. The kidneys were macroscopically unremarkable. Both adrenal glands were markedly enlarged weighing 68 and 78 gms (normal weight up to $8 \mathrm{gm}$ ) and replaced by yellowish material that microscopically showed areas of confluent necrosis and contained numerous small refractile budding yeasts 2-4 microns in diameter. These had the appearance of Histoplasma capsulatum and were stained by the Grocott stain. Mucicarmine stains were negative and the yeasts did not have the thick capsule or pleomorphism of Cryptococcus neoformans. No hyphi were seen and the cells were smaller than those of Candida albicans.

\section{Nervous System}

The brain weighed $1380 \mathrm{~g}$ after fixation and was externally unremarkable apart from a slight opacity of the leptomeninges. Coronal sections of the brain showed multiple firm discrete nodules up to $0.5 \mathrm{~cm}$ in diameter in the left temporal lobe (figure 1), left occipital lobe, right occipital lobe and the left internal capsule. A $2 \mathrm{~mm}$ diameter nodule was present in the superior surface of the right lobe of the cerebellum and a $3 \mathrm{~mm}$ nodule was present in the vermis. A four millimetre diameter nodule was present in the right anterior zone of the pons (figure $2 a$ ) and a similar lesion was found within the mid-lumbar region of the spinal cord (figure $2 \mathrm{~b}$ ). Microscopically the nodules were composed of necrotic material and contained similar refractile budding yeasts to those in the adrenal glands (figure $3 a$ and $b$ ). At the periphery of the nodules there was a chronic inflammatory cell infiltrate composed of lymphocytes and macrophages. No giant cells were identified. There was no evidence of histoplasma-like organisms in the leptomeninges or the nerve roots. No pathological examination of peripheral nervous system tissue was carried out. 


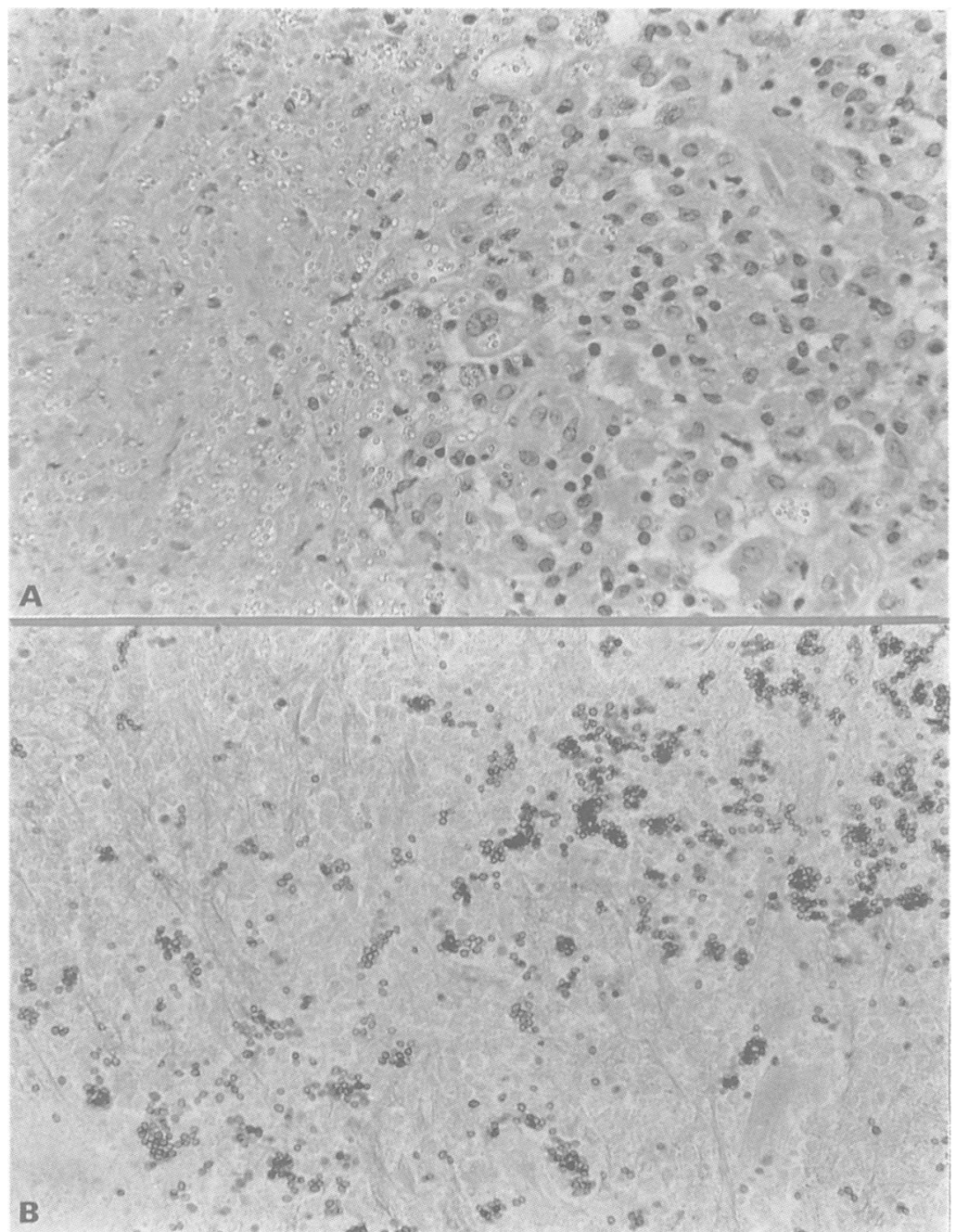

Figure 3 (a) Haematoxylin and eosin stained section from the edge of the pontine lesions showing numerous refractile particles often within macrophages. Original magnification $\times 40$. (b) Centre of the pontine lesion with organisms stained positive with Grocotts stain. Original magnification $\times 40$. disease acquired by Europeans exposed in South and South East Asia. Symmers reported on a series of 48 cases who mostly had painful mucocutaneous ulceration and a liability to acute adrenal insufficiency. Of the 23 fatal cases 11 died from adrenal failure, eight from disseminated histoplasmosis without significant adrenal or CNS involvement, two from meningo encephalitis, one from myocarditis and one from inanition. ${ }^{8-9}$

The differential diagnosis of infection due to Cryptococcus neoformans was also considered but felt to have been excluded for the following reasons: stains for mucin containing capsular material with alcian blue and mucicarmine were negative, Cryptococcus is usually more pleomorphic in shape and size than the form seen in our case, prominent adrenal gland involvement is typical of histoplasmosis but very rare in cryptococosis and finally the organisms stained clearly positive with the specific antihistoplasma antibody and negative with the cryptococcal anti-serum.

Primary neurological presentation of histoplasmosis are rare even in endemic areas although CNS involvement in disseminated cases is quite common histologically. Shulz reported involvement of the brain or meninges in $10 \%$ of a necropsy series. ${ }^{6}$ Two other series suggested CNS involvement in about a quarter of all patients with disseminated histoplasmosis. ${ }^{10-11}$ There is no recent English language review of neurological involvement with histoplasmosis. There are only single neurosurgical case reports in which isolated contrast enhancing lesions seen on CT brain scans have, after biopsy for suspected tumour or pyogenic abscess, turned out to be due to histoplasmosis. Four recent reports have involved isolated lesions, two in the cerebral hemisphere and two in the thalamus. ${ }^{12-15}$ There are no previous reports of histoplasmosis presenting as a myelopathy.

This case illustrates the increasing importance of screening all patients with unexplained CNS disease for rare infective causes and in particular patients who may have lived in endemic areas in their distant past. The conventional serological tests for histoplasmosis using immunodiffusion and complement fixation are unfortunately often negative in chronic forms of disseminated histoplasmosis but a sensitive radioimmuno assay for detecting $H$ capsulatum antigen in serum and urine now exists ${ }^{16}$ although it is not widely available. The biopsy and culture of focal lesions is often still essential.

\section{Discussion}

It was presumed that this patient acquired a primary infection with $H$ capsulatum as a young person in India which remained latent for over 35 years until he developed fibrosing alveolitis and cryptogenic cirrhosis. There was no evidence of histoplasmosis on reviewing his original liver and lung biopsy specimens. It is assumed that reactivation of his fungal disease occurred due to steroid treatment and diabetes mellitus rather than HIV infection.

Involvement of the adrenal cortex by $H$ capsulatum seems to be a particular feature of
1 Wiles CM, Mackenzie DWR. In: Kennedy PGE, Johnson $\mathrm{RT}$, eds. Infections of the nervous system. London: Butterworths, 1987.

2 Sotgin G, Mantanovi A, Mazzonia T. Histoplasmosis in Europe. Myopathologica 1970;40:53-74

3 Wheat J, Slama T, Zeckel M. Histoplasmosis in the acquired immunodeficiency syndrome. Am 7 Medicine 1985 78:203-10.

4 Bonner J, Alexander W, Dismukes W. Disseminated histoplasmosis in patients with the acquired immune deficiency syndrome. Arch Intern Med 1984;144:2178-81.

Wheat J, Small C. Disseminated histoplasmosis in the acquired immune deficiency syndrome. Arch Intern Med 6 Schula 140 Schulz DM. Histoplasmosis of the central nervous system. 
7 Shapiro JL, Lux JJ, Sprofkin BE. Histoplasmosis of the central nervous system. Am F Pathol 1955;31:319-35.

8 Symmers W St C. Histoplasmosis in Southern and South Eastern Asia. Ann Soc Belge Med Trop 1972;52:435-52.

9 Symmers W St C. Deep seated fungal infections currently seen in the histopathological series of a medical school laboratory in Britain. Amer $\mathcal{f}$ Clin Path 1966;46:514-37.

10 laboratory in Britain. Amer f Clin Path 1966;46:514-37. nervous system. Report of two cases and review of the nervous system. Report of two cases

literature. Am f Med 1963;35:45-57.
11 Smith JW, Utz LP. Progressive disseminated histoplasmosis. A prospective study of 26 patients. Ann Intern Med 1972;76:557-65.

12 Allo MDA, Silva J, Kauffman CA, Kicks RE. Enlarging histoplasmosas following treatment of meningitis due to histoplasma capsulatum. 7 Neurosurg 1979;51:242-4.

13 Vakili S, Eble J, Richmond BD, Yount RA. Cerebral histoplasmoma. $f$ Neurosurg 1983;59:332-6.

14 Dion FM, Verger BH, Landon G, Handel SF. Thalamic histoplasmoma: CT and MR imaging. $\mathcal{F}$ Comput Assist histoplasmoma: CT and

15 Venger BH, Langdon G, Rose JE. Solitary histoplasmoma of the thalamus: case report and literature review. Neurosurgery 1987;20:784-7.

16 Wheat LJ, Kohler RB, Tweari RP. Diagnosis of disseminated histoplasmosis by detection of histoplasma capsulatum antigen in serum and urine specimens. $N$ Engl $\mathcal{F} \mathrm{Med}$ 1986;314:83-8. 